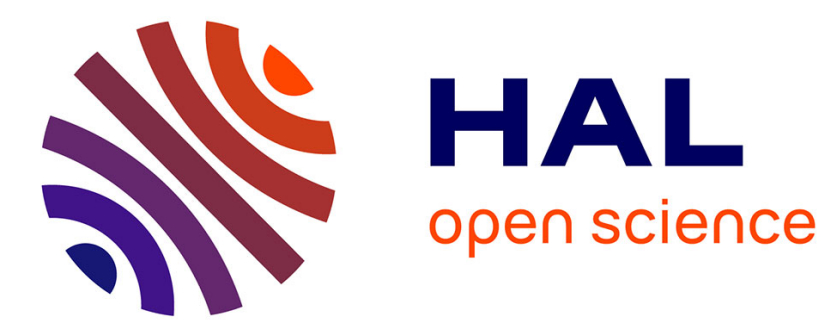

\title{
Uplink Energy Efficiency Distribution with Aerial Users in Cellular Networks
}

Yassine Hmamouche, Mustapha Benjillali, Samir Saoudi, Daniel Benevides da Costa

\section{- To cite this version:}

Yassine Hmamouche, Mustapha Benjillali, Samir Saoudi, Daniel Benevides da Costa. Uplink Energy Efficiency Distribution with Aerial Users in Cellular Networks. IEEE Wireless Communications Letters, 2021, 10 (2), 10.1109/LWC.2020.3029544 . hal-02963321

\section{HAL Id: hal-02963321 \\ https://hal.science/hal-02963321}

Submitted on 10 Oct 2020

HAL is a multi-disciplinary open access archive for the deposit and dissemination of scientific research documents, whether they are published or not. The documents may come from teaching and research institutions in France or abroad, or from public or private research centers.
L'archive ouverte pluridisciplinaire HAL, est destinée au dépôt et à la diffusion de documents scientifiques de niveau recherche, publiés ou non, émanant des établissements d'enseignement et de recherche français ou étrangers, des laboratoires publics ou privés. 


\title{
Uplink Energy Efficiency Distribution with Aerial Users in Cellular Networks
}

\author{
Yassine Hmamouche, Student Member, IEEE, Mustapha Benjillali, Senior Member, IEEE, \\ Samir Saoudi, Senior Member, IEEE, and Daniel Benevides da Costa, Senior Member, IEEE
}

\begin{abstract}
The power consumption of future user equipments (UEs) will be affected by the projected growth in their computing capacity, while data throughput may be affected by emerging aerial UEs with specific radio propagation conditions compared to terrestrial UEs. In such a context, this letter evaluates a key metric of interest, namely the probability that the uplink energy efficiency (EE) at a typical ground base station will be higher than a predefined threshold. We first characterize the priority bias of each UE layer as a function of long-term shadowing and systemlevel parameters to assess its penetration rate, i.e., the amount of active UEs from each tier among the total population of active UEs. Next, tractable approximations of the desired signal and the interference distribution are performed, enabling to derive the uplink EE. Our results demonstrate that an aggregation of the system-level parameters through the aerial priority bias needs to meet a given constraint to mitigate interference from aerial UEs and enhance the uplink EE of ground UEs. Monte-Carlo simulations validate the accuracy of our analytical results.
\end{abstract}

Index Terms-Power Control, Signal-to-Interference Ratio, Stochastic Geometry, Unmanned Aerial Vehicles (UAVs).

\section{INTRODUCTION}

Given their agility and flexible deployment, interest in unmanned aerial vehicles (UAVs) technology is rapidly growing, opening doors to various realms of application. The ongoing technological advances and upcoming generations of wireless networks such as fifth generation $(5 \mathrm{G})$ and beyond (B5G), will enable these equipments to be enhanced with many new sensors and seamless connectivity, making them more robust and more useful than their older versions.

Despite their expected benefits, UAVs as aerial user equipment (UE) in communication networks, can nevertheless have a detrimental impact on the performance of terrestrial UEs, which are often assigned more critical tasks than UAVs (e.g., monetary transactions, health-care services) [1]. The Third Generation Partnership Project (3GPP) has involved in Release 15 a technical study to assess the capability to serve aerial UEs through Long Term Evolution (LTE) deployments where base station (BS) antennas are targeting terrestrial UEs [2]. It is particularly observed from simulations that an increased density $^{1}$ of aerial UEs will significantly increase the uplink

Y. Hmamouche and S. Saoudi are with the Department of Signal and Communications, IMT-Atlantique, Brest 29200, France (e-mails: \{yassine.hmamouche, samir.saoudi\}@imt-atlantique.fr).

M. Benjillali is with the Communication Systems Department, INPT, Rabat 10100, Morocco (e-mail: benjillali@ieee.org).

D. B. da Costa is with the Department of Computer Engineering, Federal University of Ceará, Sobral 62010-560, Brazil (e-mail: danielbcosta@ieee.org).

This work was supported by Pôle de Recherche Avancée en Communications (PRACOM) and the Regional Council of Brittany, France.

${ }^{1}$ In this work, we consider an aggregated measure more general than aerial UE density, namely the aerial priority bias that we will discuss later. interference on ground BSs. This is due to the observation that a typical aerial UE experiences line-of-sight propagation conditions with a higher probability to more cells as compared to a typical terrestrial UE. This increase in uplink interference would require a higher resource utilization level to maintain a similar level of throughput for ground UEs. On the other hand, increasing the resource utilization level further magnifies the uplink interference in the network, and hence further degrades the uplink throughput of both aerial and terrestrial UEs.

Considering deterministic locations and fixed number of ground BSs, terrestrial UEs, and aerial UEs, most research efforts have generally leveraged field measurements and simulations [3], algorithmic analysis [4], and optimization theory [5] to evaluate the impact of aerial UEs on the performance of terrestrial LTE cellular networks. The above techniques are typically time-consuming, require customized setups for each experiment, and need complex and efficient algorithms. Hence, increasing need for tractable analytical models. To our knowledge, there is no analytical model available in the literature to capture the impact of UAVs system-level parameters (density, height, consumed power, and power control factor) on uplink performance metrics of cellular networks.

Stochastic geometry and its inherent point process theory is considered as a powerful mathematical tool for the systemlevel analysis of wireless networks [6]. However, uplink analysis is highly challenging due to the coupling in active UEs locations following the use of orthogonal multiple access schemes such as orthogonal frequency-division multiple access (OFDMA), and also due to distance-dependent power control schemes inducing dependency between BSs and UEs locations. Several generative models have been developed in the literature to assess the uplink performance in terrestrial single-tier cellular networks [7], multi-tier wireless networks [8], and dense cellular networks [9]. Despite these analytical models, there are still important unexplored leads that need to be addressed. For instance, i) considering heterogeneity at the UE level (terrestrial and aerial UEs) as opposed to BS level (small cells, macro cells). The uplink analysis under the latter is revealed to be statistically equivalent to that under the single tier setup [8, Corollary 4]. Also, ii) previous works have only addressed typical performance metrics such as coverage probability and throughput. Adjusting systemlevel parameters to improve these metrics would however have a detrimental impact on other important metrics such as the power consumption at UEs interested in having longer battery autonomy. Our contributions can be summarized as follows:

- We consider two classes of terrestrial and aerial UEs with distinctive parameters in terms of long-term shadowing, 
and system-level parameters, and introduce a measure of priority between UE tiers, namely the UE priority bias. The rationale is to evaluate the process of active uplink UEs from each tier and then derive the distribution of the serving UE in uplink.

- Deriving the process of interfering UEs is quite challenging in the uplink analysis. In our setup, we approximate it with an inhomogeneous Poisson point process (PPP) over an exclusion region defined by the tradeoff between interfering UEs and the serving UE average received power at the typical BS.

- Finally, we derive the distribution of the uplink energy efficiency (EE), enabling us to evaluate the amount of UEs with a good tradeoff between throughput and power consumption. Next, we illustrate the detrimental effect of increasing aerial priority bias on the EE. An analytical constraint based on the Lambert $\mathrm{W}$ function and mapping main system-level parameters is identified for proper operational regime of cellular networks with aerial UEs.

Notations: $\mathbb{P}($.$) and \mathbb{E}($.$) stand for probability and expectation$ measures. $\mathcal{L}_{X}(s)=\mathbb{E}\left\{\mathrm{e}^{-s X}\right\}$ is the Laplace transform of a random variable $X$ evaluated at $s$. For $a \in \mathbb{R}, \Gamma(a)=$ $\int_{0}^{\infty} t^{a-1} \mathrm{e}^{-t} \mathrm{~d} t$ is the Gamma function. $W_{0}$ is the principal branch of the Lambert $\mathrm{W}$ function defined by the lagrange inversion theorem as $W_{0}(x)=\sum_{n \geq 1} \frac{(-n)^{n-1}}{n !} x^{n}$.

\section{System Model}

We consider the uplink of a cellular network made of terrestrial BSs modeled according to a $2 \mathrm{D}$ homogeneous PPP $\Psi_{\mathrm{b}} \triangleq\left\{y_{i}\right\}$ with density $\lambda_{\mathrm{b}}$ in the Euclidean plane $\mathcal{P} \subset \mathbb{R}^{2}$, and a vertical set of heterogeneous UEs (having data to transmit in uplink), comprising two classes of UEs; terrestrial UEs modeled according to a HPPP $\Psi_{\mathrm{t}}$ with density $\lambda_{\mathrm{t}}$, and aerial UEs deployed at an average altitude $h_{\mathrm{a}}$ such that their projection in $\mathcal{P}$ is modeled according to a homogeneous PPP $\Psi_{\mathrm{a}}$ with density $\lambda_{\mathrm{a}}$. Without loss of generality, the typical BS $y_{0}$ at the origin $O$, is the object of the analysis.

We consider standard power-law path-loss between $y_{0}$ and UEs from $\Psi_{\mathrm{t}}$ and $\Psi_{\mathrm{a}}$ as $\ell_{\mathrm{t}}(r)=\mathrm{K} r^{-\alpha}$ and $\ell_{\mathrm{a}}(r)=$ $\mathrm{K}\left(h_{\mathrm{a}}^{2}+r^{2}\right)^{\frac{-\alpha}{2}}$, respectively, where $r$ is the horizontal distance between the UE of interest and $y_{0}, \alpha>2$ is the pathloss exponent, and $\mathrm{K}=\left(\frac{3.10^{8}}{4 \pi f}\right)^{2}$ is the free-space pathloss such that $f$ is the transmission frequency. Multipath fading of the link between $y_{0}$ and a UE $x$, is incorporated by a positive independent and identically distributed (i.i.d.) Rayleigh random variable (RV) $g_{x}$ with unit average power, i.e., $g_{x} \sim \exp (1)$. Also, for $i \in\{\mathrm{t}, \mathrm{a}\}$, the link between $y_{0}$ and a UE from $\Psi_{i}$ is subject to shadowing such that its power is modeled by log-normal RVs $\chi_{i}$ with mean $\mu_{i}$ (in $\mathrm{dB}$ ) and standard deviation $\sigma_{i}$ (in $\mathrm{dB}$ ). Based on the displacement theorem, shadowing effect can be absorbed into the HPPP density. In this way, the displaced HPPP is still noted $\Psi_{i}$ with density $\bar{\lambda}_{i}=\lambda_{i} \mathbb{E}\left(\chi_{i}^{2 / \alpha}\right)<\infty$, where $\mathbb{E}\left(\chi_{i}^{2 / \alpha}\right)=\exp \left(\frac{\ln (10)}{5} \frac{\mu_{i}}{\alpha}+\left(\frac{\ln (10)}{5 \sqrt{2}} \frac{\sigma_{i}}{\alpha}\right)^{2}\right)$.

The total power usage for the uplink transmission of a UE from $\Psi_{i}$ is $P_{\text {usage }}^{i}=P_{\mathrm{s}, i}+P_{\mathrm{d}, i}$, where $P_{\mathrm{s}, i}$ and $P_{\mathrm{d}, i}$

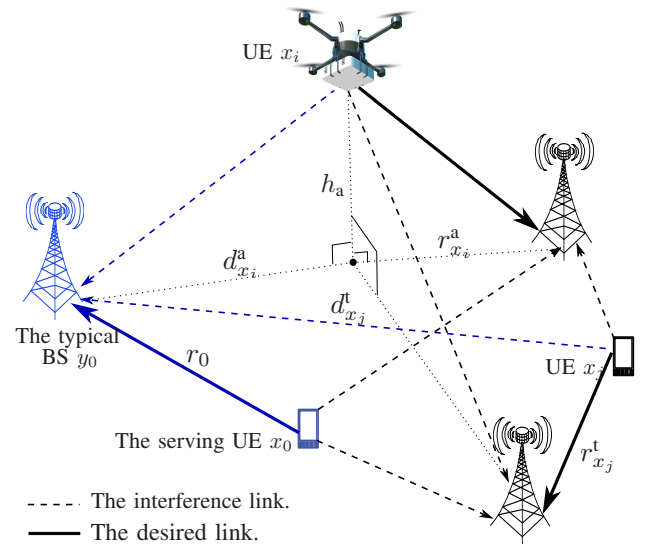

Fig. 1. PPP locations of BSs, terrestrial UEs, and aerial UEs. The BS $y_{0}$ is served by UE $x_{0}$ and jammed by signals from the other active UEs.

are, respectively, the static power consumed in UE's internal processes (e.g., signal processing, computing tasks, battery backup) and the dynamic power for wireless transmissions tuned in accordance with the distance to the associated BS since UEs are battery-powered. In this paper, we consider the fractional power control (FPC) such that $P_{\mathrm{d}, i}=P_{i}\left(\ell_{i}(r)\right)^{-\epsilon_{i}}$, where $\epsilon_{i} \in[0,1]$ is the power control factor and $P_{i}$ is the UE transmit power when no power control is considered, i.e., $\epsilon_{i}=0 . P_{i}$ can be seen as a selection bias to tune the uplink range of UEs. In a given time/frequency resource, the typical BS randomly selects a single active uplink UE from all the UEs having a vertical projection inside its Voronoi cell ${ }^{2}$, namely $\mathcal{C}_{0}$. We focus on the loaded regime where each BS is active in the uplink. After associating only one UE per each BS, we have a network with a mixture of active terrestrial and aerial UEs. The amount of active UEs from each tier, i.e., the penetration rate, can be tuned by a knob of priority bias that naturally has to be designed as a function of shadowing and all system parameters (e.g., UE density, power bias, power control factor, and altitude). For instance, we can increase the tendency of the typical BS to be connected to aerial UEs in uplink by lowering their altitude and/or increasing their density.

For tractability, we consider the following assumptions.

Assumption 1. The class of UEs with higher priority bias is the one offering the best uplink average received power.

Assumption 2. The process of active uplink UEs (those scheduled to serve their own $B S$ ) is assumed to preserve the Poisson law as was endorsed by simulations in [10, Fig. 5].

Given Assumptions 1 and 2, the priority bias measure can be used to characterize the process of active terrestrial and aerial UEs via independent thinning, respectively, denoted by $\widetilde{\Psi}_{\mathrm{t}}$ and $\widetilde{\Psi}_{\mathrm{a}}$ with respective densities $\widetilde{\lambda}_{\mathrm{t}}=\mathcal{A}_{\mathrm{t}} \lambda_{\mathrm{b}}$ and $\widetilde{\lambda}_{\mathrm{a}}=\mathcal{A}_{\mathrm{a}} \lambda_{\mathrm{b}}$, where $\mathcal{A}_{\mathrm{t}}$ and $\mathcal{A}_{\mathrm{a}}$ are the priority bias of terrestrial and aerial UEs, respectively. If terrestrial and aerial UEs are identical in terms of long-term shadowing and system-level parameters, we naturally need to get $\mathcal{A}_{\mathrm{t}}=\mathcal{A}_{\mathrm{a}}=0.5$. The following Lemma considers the general setup.

Lemma 1. The priority bias of terrestrial and aerial UEs is, respectively, expressed as

\footnotetext{
${ }^{2}$ Hence, intra-cell interference is ignored, while inter-cell interference is present due to universal frequency reuse.
} 


$$
\begin{aligned}
& \mathcal{A}_{t}=1-\exp \left(-\pi \bar{\lambda}_{t} \delta^{2}\right)+2 \pi \bar{\lambda}_{t} \mathrm{e}^{\pi \bar{\lambda}_{a} h_{a}^{2}} \\
& \times \int_{\delta}^{\infty} r \exp \left(-\pi\left(\bar{\lambda}_{t} r^{2}+\bar{\lambda}_{a}\left(\mathrm{~K}^{\epsilon_{t}-\epsilon_{a}} \frac{P_{a}}{P_{t}}\right)^{\frac{2}{\alpha\left(1-\epsilon_{a}\right)}} r^{\frac{2\left(1-\epsilon_{t}\right)}{1-\epsilon_{a}}}\right)\right) \mathrm{d} r
\end{aligned}
$$

$\mathcal{A}_{a}=2 \pi \bar{\lambda}_{a}$

$\times \int_{0}^{\infty} r \exp \left(-\pi\left(\bar{\lambda}_{a} r^{2}+\bar{\lambda}_{t}\left(\mathrm{~K}^{\epsilon_{a}-\epsilon_{t}} \frac{P_{t}}{P_{a}}\right)^{\frac{2}{\alpha\left(1-\epsilon_{t}\right)}}\left(r^{2}+h_{a}^{2}\right)^{\frac{1-\epsilon_{a}}{1-\epsilon_{t}}}\right)\right) \mathrm{d} r$,

where $\delta=h_{a}^{\frac{1-\epsilon_{a}}{1-\epsilon_{t}}}\left(\mathrm{~K}^{\epsilon_{a}-\epsilon_{t}} \frac{P_{t}}{P_{a}}\right)^{\frac{1}{\alpha\left(1-\epsilon_{t}\right)}}$.

Proof. For $i \in\{\mathrm{t}, \mathrm{a}\}$, the complementary cumulative distribution function (CCDF) of $\overline{r_{i}}$, the smallest distance to contact a UE from $\Psi_{i}$, is obtained from the null probability of the PPP $\Psi_{i}$ as

$$
F_{\overline{r_{i}}}(u)=\mathbb{P}\left(\bar{r}_{i}>u\right)=\exp \left(-\pi \bar{\lambda}_{i} u^{2}\right) .
$$

From the abstraction of $\mathcal{A}_{\mathrm{t}}$ in Assumption 1, we get

$$
\begin{aligned}
& \mathcal{A}_{\mathrm{t}}=\mathbb{P}\left(\mathrm{K}^{1-\epsilon_{\mathrm{t}}} P_{\mathrm{t}} \overline{r_{\mathrm{t}}}-\alpha\left(1-\epsilon_{\mathrm{t}}\right) \geq \mathrm{K}^{1-\epsilon_{\mathrm{a}}} P_{\mathrm{a}}\left({\overline{r_{\mathrm{a}}}}^{2}+h_{\mathrm{a}}^{2}\right)^{-\frac{\alpha\left(1-\epsilon_{\mathrm{a}}\right)}{2}}\right) \\
& =\mathbb{E}_{\overline{r_{\mathrm{t}}}}\left(\mathbb{P}\left({\overline{r_{\mathrm{a}}}}^{2} \geq\left(\mathrm{K}^{\epsilon_{\mathrm{t}}-\epsilon_{\mathrm{a}}} \frac{P_{\mathrm{a}}}{P_{\mathrm{t}}}\right)^{\frac{2}{\alpha\left(1-\epsilon_{\mathrm{a}}\right)}} u^{\frac{2\left(1-\epsilon_{\mathrm{t}}\right)}{1-\epsilon_{\mathrm{a}}}}-h_{\mathrm{a}}^{2} \mid \overline{r_{\mathrm{t}}}=u\right)\right) \\
& =-\int_{0}^{\infty} F_{\overline{r_{\mathrm{a}}}}\left(\sqrt{\max \left(0,\left(\mathrm{~K}^{\epsilon_{\mathrm{t}}-\epsilon_{\mathrm{a}}} \frac{P_{\mathrm{a}}}{P_{\mathrm{t}}}\right)^{\frac{2}{\alpha\left(1-\epsilon_{\mathrm{a}}\right)}} u^{\frac{2\left(1-\epsilon_{\mathrm{t}}\right)}{1-\epsilon_{\mathrm{a}}}}-h_{\mathrm{a}}^{2}\right)}\right) \mathrm{d} F_{\overline{r_{\mathrm{t}}}}(u) .
\end{aligned}
$$

Exact expression of $\mathcal{A}_{\mathrm{t}}$ is then obtained by using CCDFs in (3) and splitting the interval of integration based on $\delta$. A similar approach is followed to derive $\mathcal{A}_{\mathrm{a}}$.

Special cases: when $\epsilon_{\mathrm{t}}=\epsilon_{\mathrm{a}}=\epsilon$, Lemma 1 can be simplified under a closed-form expression as

$$
\begin{aligned}
& \mathcal{A}_{\mathrm{a}}=1-\mathcal{A}_{\mathrm{t}} \\
& =\frac{\bar{\lambda}_{\mathrm{a}} P_{\mathrm{a}}^{\frac{2}{\alpha(1-\epsilon)}}}{\bar{\lambda}_{\mathrm{t}} P_{\mathrm{t}}^{\frac{2}{\alpha(1-\epsilon)}}+\bar{\lambda}_{\mathrm{a}} P_{\mathrm{a}}^{\frac{2}{\alpha(1-\epsilon)}}} \exp \left(-\pi \bar{\lambda}_{\mathrm{t}} h_{\mathrm{a}}^{2}\left(\frac{P_{\mathrm{t}}}{P_{\mathrm{a}}}\right)^{\frac{2}{\alpha(1-\epsilon)}}\right) .
\end{aligned}
$$

If we also have $P_{\mathrm{a}}=P_{\mathrm{t}}$, (8) can be further simplified as

$$
\mathcal{A}_{\mathrm{a}}=1-\mathcal{A}_{\mathrm{t}}=\frac{\bar{\lambda}_{\mathrm{a}}}{\bar{\lambda}_{\mathrm{t}}+\bar{\lambda}_{\mathrm{a}}} \exp \left(-\pi \bar{\lambda}_{\mathrm{t}} h_{\mathrm{a}}^{2}\right) .
$$

For $i \in\{\mathrm{t}, \mathrm{a}\}$, we denote by $r_{x}^{i}$ the horizontal distance between a given UE $x \in \widetilde{\Psi}_{i}$ and its nearest BS. Particularly, $r_{\mathrm{t}}$ and $r_{\mathrm{a}}$ are, respectively, the horizontal distance from $y_{0}$ to its nearest UE from $\widetilde{\Psi}_{\mathrm{t}}$ and $\widetilde{\Psi}_{\mathrm{a}}$. Also, we denote by $\mathcal{S}$ the serving tier, i.e., $\mathcal{S}=\widetilde{\Psi}_{\mathrm{t}}$ or $\mathcal{S}=\widetilde{\Psi}_{\mathrm{a}}$, and by $r_{0}$ the horizontal distance from $y_{0}$ to its serving UE $x_{0} \in \mathcal{S}$. The distance from $y_{0}$ to an interfering UE $x$ from $\widetilde{\Psi}_{i} \backslash\left\{x_{0}\right\}$ is denoted by $d_{x}^{i}$. Fig. 1 illustrates the setup of our system model.

For the sake of simplicity, we ignore thermal noise and focus our analysis on the interference-limited regime [7], [8]. In such a context, the signal-to-interference ratio (SIR) at $y_{0}$ is given by

$$
\operatorname{SIR}\left(x_{0} \in \widetilde{\Psi}_{i} ; y_{0}\right)=\frac{g_{x_{0}} P_{i}\left(\ell_{i}\left(r_{0}\right)\right)^{1-\epsilon_{i}}}{I_{x_{0}, i}^{\mathrm{t}}+I_{x_{0}, i}^{\mathrm{a}}},
$$

where for $i, j \in\{t, a\}, I_{x_{0}, i}^{j}$ is the interference generated by UEs from $\widetilde{\Psi}_{j} \backslash\left\{x_{0}\right\}$ conditioned on a serving UE $x_{0} \in \widetilde{\Psi}_{i}$. It is expressed as

$$
I_{x_{0}, i}^{j}=\sum_{x \in \widetilde{\Psi}_{j} \backslash\left\{x_{0} \in \widetilde{\Psi}_{i}\right\}} g_{x} P_{j} \ell_{j}\left(d_{x}^{j}\right)\left(\ell_{j}\left(r_{x}^{j}\right)\right)^{-\epsilon_{j}} .
$$

\section{UPLINK ENERGY EFFICIENCY DISTRIBUTION}

In this section, we provide the key analytical framework to derive the uplink EE distribution under a setup with terrestrial and aerial UEs.

Definition 1. The uplink rate (in bps) at the typical $B S y_{0}$ when it is served by $x_{0} \in \widetilde{\Psi}_{i}(i \in\{t, a\})$ is

$$
\mathcal{R}_{i}=\frac{\mathrm{B}}{\mathrm{N}+1} \log _{2}\left(1+\operatorname{SIR}\left(x_{0} \in \widetilde{\Psi}_{i} ; y_{0}\right)\right),
$$

where $\mathrm{B}$ is the total effective uplink bandwidth in $\mathrm{Hz}$ and $\mathrm{N}$ is the total number of UEs from $\Psi_{t} \cup \Psi_{a} \backslash\left\{x_{0} \in \widetilde{\Psi}_{i}\right\}$ with orthogonal projection inside $\mathcal{C}_{0}$.

Definition 2. (Energy efficiency coverage). EE coverage $\mathcal{E}_{c}$ is defined as the probability that the uplink EE measured at the typical BS is higher than a predefined threshold T. Formally,

$$
\mathcal{E}_{c}=\sum_{i \in\{t, a\}} \mathbb{P}\left(\frac{\mathcal{R}_{i}}{P_{s, i}+P_{d, i}} \geq \mathrm{T}, \mathcal{S}=\widetilde{\Psi}_{i}\right) .
$$

The EE distribution is completely characterized by the EE coverage. Also, $\mathrm{N}$ is a $\mathrm{RV}$ depending on the serving area of the typical BS $y_{0}$, i.e., $\mathcal{C}_{0}$, and the priority bias that governs the number of active terrestrial and aerial UEs in the uplink.

Assumption 3. Given the orthogonal allocation of channel resources, it is reasonable to consider that the number $\mathrm{N}$ of competing UEs is uncorrelated with SIR at $y_{0}$.

In this way, the EE coverage can be derived as

$\mathcal{E}_{\mathrm{c}}=\sum_{i \in\{\mathrm{t}, \mathrm{a}\}} \mathbb{P}\left(\frac{\log _{2}\left(1+\operatorname{SIR}\left(x_{0} \in \widetilde{\Psi}_{i} ; y_{0}\right)\right)}{P_{\mathrm{s}, i}+P_{\mathrm{d}, i}} \geq \frac{\mathrm{T}(\mathrm{N}+1)}{\mathrm{B}}, \mathcal{S}=\widetilde{\Psi}_{i}\right)$

$\stackrel{(\mathrm{a})}{=} \sum_{m \geq 0} \sum_{i \in\{\mathrm{t}, \mathrm{a}\}} \mathbb{P}(\mathrm{N}=m) \mathbb{P}\left(g_{x_{0}} \geq s_{i}\left(m, r_{0}\right)\left(I_{x_{0}, i}^{\mathrm{t}}+I_{x_{0}, i}^{\mathrm{a}}\right), \mathcal{S}=\widetilde{\Psi}_{i}\right)$

$\stackrel{\text { (b) }}{=} \sum_{m \geq 0} \sum_{i \in\{\mathrm{t}, \mathrm{a}\}} \mathbb{P}(\mathrm{N}=m) \mathbb{E}_{r_{0}}\left(\mathcal{L}_{I_{x_{0}, i}^{\mathrm{t}}}\left(s_{i}\left(m, r_{0}\right)\right) \mathcal{L}_{I_{x_{0}, i}^{\mathrm{a}}}\left(s_{i}\left(m, r_{0}\right)\right)\right)$,

where (a) comes from the independence between the distribution of the number of competing UEs to $x_{0}$ and the received SIR from $x_{0}$, (b) comes from $g_{x} \sim \exp (1)$ and the definition of the Laplace functional, where for $i \in\{\mathrm{t}, \mathrm{a}\}$, $s_{i}\left(m, r_{0}\right)=\frac{2^{\frac{\mathrm{T}(m+1)}{\mathrm{B}}\left(P_{\mathrm{s}, i}+P_{i}\left(\ell_{i}\left(r_{0}\right)\right)^{-\epsilon_{i}}\right)}-1}{P_{i}\left(\ell_{i}\left(r_{0}\right)\right)^{1-\epsilon_{i}}}$, and the probability mass function (PMF) of $\mathrm{N}$ is [11]

$\mathbb{P}(\mathrm{N}=m) \simeq \frac{3.5^{3.5}}{\Gamma(3.5)} \frac{\Gamma(m+4.5)}{m !}\left(\frac{\bar{\lambda}_{\mathrm{t}}+\bar{\lambda}_{\mathrm{a}}}{\lambda_{\mathrm{b}}}\right)^{m}\left(3.5+\frac{\bar{\lambda}_{\mathrm{t}}+\bar{\lambda}_{\mathrm{a}}}{\lambda_{\mathrm{b}}}\right)^{-(m+4.5)}$

We derive next the supplementary terms for the computation of the uplink EE coverage. 


\section{A. Distribution of the serving UE}

After associating one UE per each BS, the probability density function (PDF) of the horizontal distance between the typical BS $y_{0}$ and its serving UE $x_{0}$ from $\widetilde{\Psi}_{\mathrm{t}}$ is derived as

$$
\begin{aligned}
& f_{r_{0}}\left(u, \mathcal{S}=\widetilde{\Psi}_{\mathrm{t}}\right)=f_{r_{\mathrm{t}}}(u) \\
& \times \mathbb{P}\left(\mathrm{K}^{-\epsilon_{\mathrm{t}}} P_{\mathrm{t}} u^{-\alpha\left(1-\epsilon_{\mathrm{t}}\right)}>\mathrm{K}^{-\epsilon_{\mathrm{a}}} P_{\mathrm{a}}\left(r_{\mathrm{a}}^{2}+h_{\mathrm{a}}^{2}\right)^{-\frac{\alpha\left(1-\epsilon_{\mathrm{a}}\right)}{2}}\right) \\
& =2 \pi \widetilde{\lambda}_{\mathrm{t}} u \exp \left(-\pi \widetilde{\lambda}_{\mathrm{t}} u^{2}\right) \\
& \times\left\{\exp \left(-\pi \widetilde{\lambda}_{\mathrm{a}}\left(\left(\mathrm{K}^{\epsilon_{\mathrm{t}}-\epsilon_{\mathrm{a}}} \frac{P_{\mathrm{a}}}{P_{\mathrm{t}}}\right)^{\frac{2}{\alpha\left(1-\epsilon_{\mathrm{a}}\right)}} u^{\frac{2\left(1-\epsilon_{\mathrm{t}}\right)}{1-\epsilon_{\mathrm{a}}}}-h_{\mathrm{a}}^{2}\right)\right),\right. \text { otherwise. }
\end{aligned}
$$

Similarly, the PDF of the horizontal distance between the typical BS $y_{0}$ and its serving UE $x_{0}$ from $\widetilde{\Psi}_{\mathrm{a}}$ is

$$
\begin{aligned}
& f_{r_{0}}\left(u, \mathcal{S}=\widetilde{\Psi}_{\mathrm{a}}\right)=f_{r_{\mathrm{a}}}(u) \\
& \times \mathbb{P}\left(\mathrm{K}^{-\epsilon_{\mathrm{a}}} P_{\mathrm{a}}\left(u^{2}+h_{\mathrm{a}}^{2}\right)^{-\frac{\alpha\left(1-\epsilon_{\mathrm{a}}\right)}{2}}>\mathrm{K}^{-\epsilon_{\mathrm{t}}} P_{\mathrm{t}} r_{\mathrm{t}}^{-\alpha\left(1-\epsilon_{\mathrm{t}}\right)}\right) \\
& =2 \pi \widetilde{\lambda}_{\mathrm{a}} u \exp \left(-\pi \widetilde{\lambda}_{\mathrm{a}} u^{2}\right) \\
& \times \exp \left(-\pi \widetilde{\lambda}_{\mathrm{t}}\left(\left(\mathrm{K}^{\epsilon_{\mathrm{a}}-\epsilon_{\mathrm{t}}} \frac{P_{\mathrm{t}}}{P_{\mathrm{a}}}\right)^{\frac{2}{\alpha\left(1-\epsilon_{\mathrm{t}}\right)}}\left(u^{2}+h_{\mathrm{a}}^{2}\right)^{\frac{1-\epsilon_{\mathrm{a}}}{1-\epsilon_{\mathrm{t}}}}\right)\right) .
\end{aligned}
$$

The above expressions of the serving distance PDF are different from those defined in [7]-[9], where only one homogeneous class of UEs is considered.

Approximating the process of interfering UEs is quite challenging due to coupling in UEs locations. For tractability, we adopt a similar abstraction as in [8], [10], where for $i \in\{\mathbf{t}, \mathbf{a}\}$,

Assumption 4. The process of interferers from $\widetilde{\Psi}_{i}$, conditioned on the serving $U E$, is modeled by an inhomogeneous PPP of density $\widetilde{\lambda}_{i}\left(1-\exp \left(-\pi \widetilde{\lambda}_{i} v^{2}\right)\right)$, where $v$ is the horizontal distance to a given interferer.

Assumption 5. Conditioned on an interfering $U E x \in \widetilde{\Psi}_{i}$, the PDF of $r_{x}^{i}$ is expresssed under a truncated version as

$$
f_{r_{x}^{i}}\left(w \mid d_{x}^{i}=v\right)=\frac{2 \pi \widetilde{\lambda}_{i} w \exp \left(-\pi \widetilde{\lambda}_{i} w^{2}\right)}{1-\exp \left(-\pi \widetilde{\lambda}_{i} v^{2}\right)}, \forall w \leq v .
$$

The exclusion region of interfering UEs under our setup is defined by the observation that the average received power from any interfering UE at $y_{0}$ needs to be lower than that received from the serving UE $x_{0}$. Otherwise, the interfering UE will be associated to the typical BS $y_{0}$. Formally,

$$
P_{i} \ell_{i}\left(d_{x}^{i}\right)\left(\ell_{i}\left(r_{x}^{i}\right)\right)^{-\epsilon_{i}} \leq P_{j}\left(\ell_{j}\left(r_{0}\right)\right)^{1-\epsilon_{j}}, \forall i, j \in\{\mathrm{t}, \mathrm{a}\} .
$$

\section{B. Interference from active terrestrial users}

For $j \in\{\mathbf{t}, \mathbf{a}\}$, the Laplace functional of the interference generated by UEs from $\widetilde{\Psi}_{\mathrm{t}}$ when the serving UE belongs to
$\widetilde{\Psi}_{j}$, is obtained as

$$
\begin{aligned}
& \mathcal{L}_{I_{x_{0}, j}^{\mathrm{t}}}(s)=\mathbb{E}\left(\exp \left(\sum_{x \in \widetilde{\Psi}_{\mathrm{t}} \backslash\left\{x_{0} \in \widetilde{\Psi}_{j}\right\}} g_{\mathrm{t}} \mathrm{K}^{1-\epsilon_{\mathrm{t}}}\left(d_{x}^{\mathrm{t}}\right)^{-\alpha}\left(r_{x}^{\mathrm{t}}\right)^{\alpha \epsilon_{\mathrm{t}}}\right)\right) \\
& =\mathbb{E}\left(\prod_{x \in \widetilde{\Psi}_{\mathrm{t}} \backslash\left\{x_{0} \in \widetilde{\Psi}_{j}\right\}} \mathbb{E}_{r_{x}^{\mathrm{t}}}\left(\frac{1}{1+s P_{\mathrm{t}} \mathrm{K}^{1-\epsilon_{\mathrm{t}}}\left(d_{x}^{\mathrm{t}}\right)^{-\alpha}\left(r_{x}^{\mathrm{t}}\right)^{\alpha \epsilon_{\mathrm{t}}}}\right)\right) \\
& \stackrel{(a)}{=} \exp \left(-4 \pi^{2} \widetilde{\lambda}_{\mathrm{t}}^{2} \int_{0}^{\infty} \int_{0}^{v} \frac{v w \mathrm{e}^{-\pi \tilde{\lambda}_{\mathrm{t}} w^{2}}}{1+\frac{K_{\mathrm{t}}-1}{s P_{\mathrm{t}}} \frac{v^{\alpha}}{w^{\alpha \epsilon_{\mathrm{t}}}}} \mathrm{d} w \mathrm{~d} v\right) \text {, }
\end{aligned}
$$

where (a) is by averaging first over the PDF of $r_{x}^{\mathrm{t}}$ in Assumption 5. Next, we use the probability generating functional (PGFL) theorem where the density of the interference field is defined in Assumption 4. The integration range of $d_{x}=v$ starts from 0 , since the closest interfering UE can be closer to $y_{0}$ than the desired UE $x_{0}$. This is possible from (23) since, for an interferer UE from $\widetilde{\Psi}_{\mathrm{t}}$ with a distance to its closest BS of $r_{x}^{\mathrm{t}}=r_{0} / n(n>1)$, it can be closer to $y_{0}$ by $r_{0} / n^{\epsilon_{\mathrm{t}}}$.

\section{Interference from active aerial users}

The Laplace functional of the interference generated by UEs from $\widetilde{\Psi}_{\mathrm{a}}$ when the serving UE belongs to $\widetilde{\Psi}_{\mathrm{t}}$ and distant from $y_{0}$ by $r_{0}=u$, is obtained as

$\mathcal{L}_{I_{x_{0}, \mathrm{t}}^{\mathrm{a}}}(s)=\exp \left(-4 \pi^{2} \widetilde{\lambda}_{\mathrm{a}}^{2} \int_{\varpi(u)}^{\infty} \int_{0}^{v} \frac{v w \mathrm{e}^{-\pi \widetilde{\lambda}_{\mathrm{a}} w^{2}}}{1+\frac{\kappa_{\mathrm{a}}-1}{s P_{\mathrm{a}}} \frac{\left(v^{2}+h_{\mathrm{a}}^{2}\right)^{\frac{\alpha}{2}}}{\left(w^{2}+h_{\mathrm{a}}^{2}\right)^{\frac{\alpha \epsilon_{\mathrm{a}}}{2}}}} \mathrm{~d} w \mathrm{~d} v\right)$,

where the starting point of the integration range with respect to $d_{x}=v$ is derived from (23) as

$$
\varpi(u)=\left\{\begin{array}{cl}
0 & , u<\delta \\
h_{\mathrm{a}} \sqrt{\left(\frac{P_{\mathrm{a}}}{P_{\mathrm{t}}} \epsilon_{\mathrm{t}}-\epsilon_{\mathrm{a}}\right)^{\frac{2}{\alpha}} \frac{u^{2\left(1-\epsilon_{\mathrm{t}}\right)}}{h_{\mathrm{a}}^{2\left(1-\epsilon_{\mathrm{a}}\right)}}-1} & , \text { otherwise. }
\end{array}\right.
$$

Similarly, the Laplace functional of the interference generated by UEs from $\widetilde{\Psi}_{\mathrm{a}}$ when the serving UE belongs to $\widetilde{\Psi}_{\mathrm{a}}$ and distant from $y_{0}$ by $r_{0}=u$, is obtained as

$\mathcal{L}_{I_{x_{0}, \mathrm{a}}^{\mathrm{a}}}(s)=\exp \left(-4 \pi^{2} \widetilde{\lambda}_{\mathrm{a}}^{2} \int_{\pi(u)}^{\infty} \int_{0}^{v} \frac{v w \mathrm{e}^{-\pi \widetilde{\lambda}_{\mathrm{a}} w^{2}}}{1+\frac{K_{\mathrm{a}}-1}{s P_{\mathrm{a}}} \frac{\left(v^{2}+h_{\mathrm{a}}^{2}\right)^{\frac{\alpha}{2}}}{\left(w^{2}+h_{\mathrm{a}}^{2}\right)^{\frac{\alpha \epsilon_{\mathrm{a}}}{2}}}} \mathrm{~d} w \mathrm{~d} v\right)$,

where $\pi(u)=h_{\mathrm{a}} \sqrt{\left(\frac{u^{2}}{h_{\mathrm{a}}^{2}}+1\right)^{1-\epsilon_{\mathrm{a}}}-1}$.

\section{NUMERICAL RESULtS}

We consider a typical cellular network where each BS has an average of 80 smartphones and 20 quadcopter UAVs within its coverage area, i.e., $\lambda_{\mathrm{t}}=80 \lambda_{\mathrm{b}}$ and $\lambda_{\mathrm{a}}=20 \lambda_{\mathrm{b}}$. We have the following standard parameters: $\alpha=4, f=2.1 \mathrm{GHz}$, $\mathrm{B}=5 \mathrm{MHz}, \mu_{\mathrm{t}}=\mu_{\mathrm{a}}=0 \mathrm{~dB}, P_{\mathrm{t}}=33 \mathrm{dBm}, P_{\mathrm{a}}=36 \mathrm{dBm}$, $P_{\mathrm{s}, \mathrm{t}}=0.5$ Watts, and $P_{\mathrm{s}, \mathrm{a}}=2.5$ Watts.

We first need to validate the expression of priority bias derived in Lemma 1. Fig. 2 illustrating the simulated and 


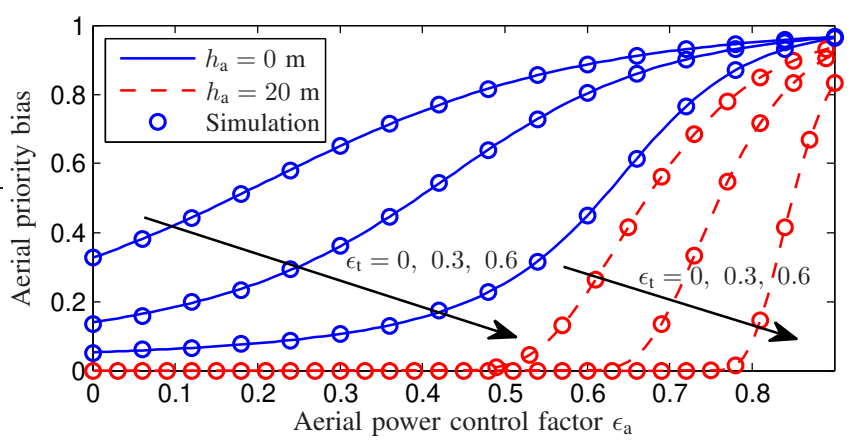

Fig. 2. Aerial priority bias as a function of terrestrial and aerial power control factors and UAVs altitude, when $\lambda_{\mathrm{b}}=10^{-2}, \sigma_{\mathrm{t}}=4 \mathrm{~dB}$, and $\sigma_{\mathrm{a}}=8 \mathrm{~dB}$.

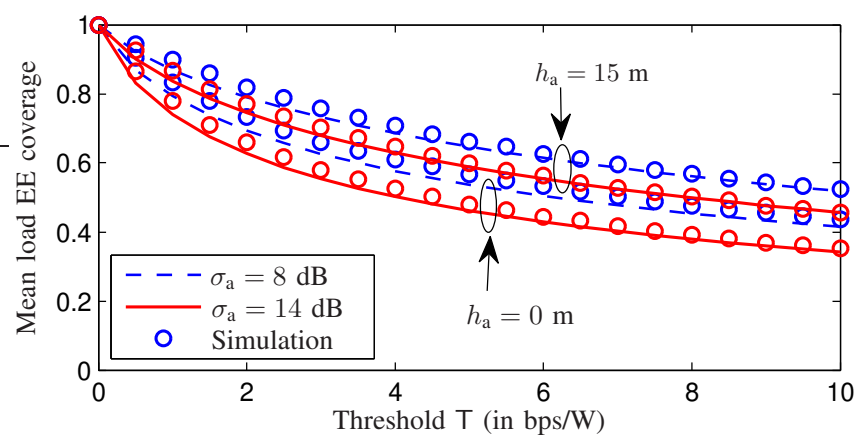

Fig. 3. Mean load approximation of the uplink EE coverage when $\lambda_{\mathrm{b}}=$ $10^{-2}, \epsilon_{\mathrm{t}}=\epsilon_{\mathrm{a}}=0.5$, and $\sigma_{\mathrm{t}}=0 \mathrm{~dB}$.

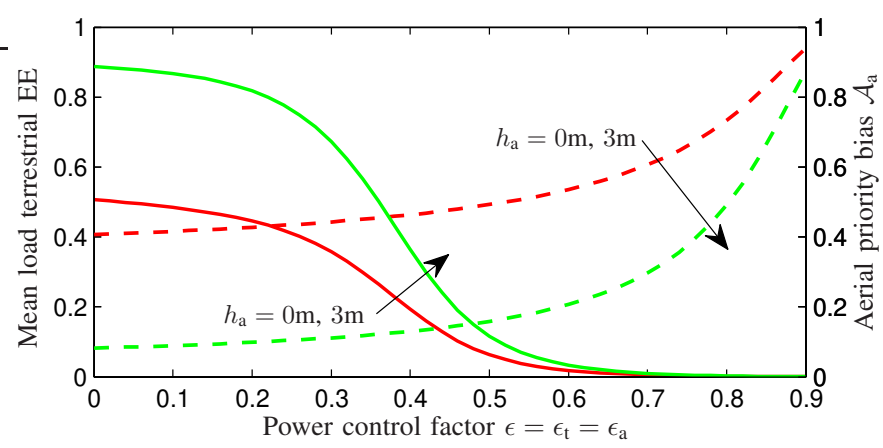

Fig. 4. Mean load approximation of the uplink terrestrial EE coverage $\mathcal{E}_{\mathrm{c}}\left(\mathcal{S}=\widetilde{\Psi}_{\mathrm{t}}\right)$ (Solid lines) and aerial priority bias $\mathcal{A}_{\mathrm{a}}$ (Dashed lines) as a function of the power control factor $\epsilon=\epsilon_{\mathrm{t}}=\epsilon_{\mathrm{a}}$ and UAVs height $h_{\mathrm{a}}$ (Arrow) when $\lambda_{\mathrm{b}}=10^{-3}, \sigma_{\mathrm{t}}=0 \mathrm{~dB}, \sigma_{\mathrm{a}}=10 \mathrm{~dB}$, and $\mathrm{T}=10 \mathrm{bps} / \mathrm{W}$.

analytical expression of the aerial priority bias shows that the analytical expression in Lemma 1 is perfectly accurate.

In Fig. 3, we validate the analytical expression of the EE coverage under the mean load setup, i.e., $\mathbb{P}\left(\mathrm{N}=1+1.28 \frac{\bar{\lambda}_{\mathrm{t}}+\bar{\lambda}_{\mathrm{a}}}{\lambda_{\mathrm{b}}}\right) \simeq 1$ [11]. It has been shown that the EE coverage decays when lowering UAVs height or increasing their density by making aerial shadowing more variable, i.e., increasing $\sigma_{\mathrm{a}}$. This is a similar result to [11], where shadowing is revealed to be a natural load balancing bias between tiers of BSs in the downlink. Here, it plays the role of a natural priority balancing between several classes of uplink UEs.

In Fig. 4, we illustrate the mean load terrestrial EE coverage, i.e., $\mathcal{E}_{\mathrm{c}}\left(\mathcal{S}=\widetilde{\Psi}_{\mathrm{t}}\right)$, and the aerial priority bias $\mathcal{A}_{\mathrm{a}}$ as a function of the power control factor $\epsilon=\epsilon_{\mathrm{t}}=\epsilon_{\mathrm{a}}$ and UAVs height $h_{\mathrm{a}}$. $\mathcal{E}_{\mathrm{c}}\left(\mathcal{S}=\widetilde{\Psi}_{\mathrm{t}}\right)$ is typically monotonically decreasing with the aerial priority bias. Reducing the latter can therefore reduce the detrimental effect of UAVs on the EE of terrestrial UEs. To achieve this, we can act not only by reducing UAVs density (as mentioned in [2]), but also by acting on other system parameters such as altitude and the power control factor (see Fig. 2). In the general case, assuming a properly operating LTE cellular network for $\mathcal{A}_{\mathrm{a}} \leq \beta<1$, we get from (8)

$$
\begin{aligned}
& \pi h_{\mathrm{a}}^{2}\left(\bar{\lambda}_{\mathrm{a}}+\bar{\lambda}_{\mathrm{t}}\left(\frac{P_{\mathrm{t}}}{P_{\mathrm{a}}}\right)^{\frac{2}{\alpha(1-\epsilon)}}\right) \exp \left(\pi h_{\mathrm{a}}^{2}\left(\bar{\lambda}_{\mathrm{a}}+\bar{\lambda}_{\mathrm{t}}\left(\frac{P_{\mathrm{t}}}{P_{\mathrm{a}}}\right)^{\frac{2}{\alpha(1-\epsilon)}}\right)\right) \\
& \geq \frac{\pi \bar{\lambda}_{\mathrm{a}} h_{\mathrm{a}}^{2}}{\beta} \exp \left(\pi \bar{\lambda}_{\mathrm{a}} h_{\mathrm{a}}^{2}\right) .
\end{aligned}
$$

Since $\mathrm{W}_{0}$ is the inverse relation of $f(w)=w \mathrm{e}^{w}$, we obtain from (30) a transcendental constraint as

$$
\left(\frac{P_{\mathrm{t}}}{P_{\mathrm{a}}}\right)^{\frac{2}{\alpha(1-\epsilon)}} \geq \frac{W_{0}\left(\frac{\pi \bar{\lambda}_{\mathrm{a}} h_{\mathrm{a}}^{2}}{\beta} \exp \left(\pi \bar{\lambda}_{\mathrm{a}} h_{\mathrm{a}}^{2}\right)\right)-\pi \bar{\lambda}_{\mathrm{a}} h_{\mathrm{a}}^{2}}{\pi \bar{\lambda}_{\mathrm{t}} h_{\mathrm{a}}^{2}} .
$$

This paper proposed a tractable analytical framework to derive the uplink EE coverage under a setup where two classes of terrestrial and aerial UEs are considered. It has been particularly shown that increased tendency of terrestrial BSs to be connected to aerial UEs, i.e., increased aerial priority bias (not only UAVs density as reported in [2]), will have a detrimental effect on the uplink EE of ground UEs. Assuming similar power control factors for terrestrial and aerial UEs, we have identified a compact analytical constraint for a proper operational regime of the network in terms of EE coverage.

Future generalization of this work will consider constrained transmit power at the level of each UE class, in addition to multi-tier BSs. The rationale is to investigate the impact of a decoupled uplink and downlink association scheme on EE.

\section{REFERENCES}

[1] B. Van Der Bergh, A. Chiumento, and S. Pollin, "LTE in the sky: trading off propagation benefits with interference costs for aerial nodes," IEEE Commun. Mag., vol. 54, no. 5, pp. 44-50, May 2016.

[2] 3GPP-TR-36.777, "Study on enhanced LTE support for aerial vehicles," 2017, 3GPP technical report. [Online]. Available: www.3gpp.org/ dynareport/36777.htm

[3] R. Amorim et al., "Measured uplink interference caused by aerial vehicles in LTE cellular networks," IEEE Wireless Commun. Lett., vol. 7, no. 6, pp. 958-961, Dec. 2018.

[4] W. Mei, Q. Wu, and R. Zhang, "Cellular-connected UAV: uplink association, power control and interference coordination," IEEE Trans. Wireless Commun., vol. 18, no. 11, pp. 5380-5393, Nov. 2019.

[5] L. Liu, S. Zhang, and R. Zhang, "Multi-beam UAV communication in cellular uplink: cooperative interference cancellation and sum-rate maximization," IEEE Trans. Wireless Commun., vol. 18, no. 10, pp. 4679-4691, Oct. 2019.

[6] J. G. Andrews, F. Baccelli, and R. K. Ganti, "A tractable approach to coverage and rate in cellular networks," IEEE Trans. Commun., vol. 59, no. 11, pp. 3122-3134, Nov. 2011.

[7] T. Novlan, H. Dhillon, and J. Andrews, "Analytical modeling of uplink cellular networks," IEEE Trans. Wireless Commun., vol. 12, no. 6, pp. 2669-2679, Jun. 2013.

[8] S. Singh, X. Zhang, and J. G. Andrews, "Uplink rate distribution in heterogeneous cellular networks with power control and load balancing," in Proc. IEEE Int. Conf. Commun. (ICC) Workshops, pp. 1275-1280, London, UK, 2015.

[9] T. Ding, M. Ding, G. Mao, Z. Lin, D. López-Pérez, and A. Y. Zomaya, "Uplink performance analysis of dense cellular networks With LoS and NLoS transmissions," IEEE Trans. Wireless Commun., vol. 16, no. 4, pp. 2601-2613, Apr. 2017.

[10] J. G. Andrews, A. K. Gupta, and H. S. Dhillon, "A primer on cellular network analysis using stochastic geometry," arXiv preprint arXiv:1604.03183, Apr. 2016.

[11] H. S. Dhillon and J. G. Andrews, "Downlink rate distribution in heterogeneous cellular networks under generalized cell selection," IEEE Wireless Commun. Lett., vol. 3, no. 1, pp. 42-45, Feb. 2014. 